\title{
Zur Kenntnis der Kondensationsprodukte von Dibenzylketon und Benzaldehyd
}

von

\begin{abstract}
Guido Goldschmiedt, w. M. k. Akad., und Karl Spitzauer.
\end{abstract}
Aus dem chemischen Laboratorium der k. k. deutschen Universität in Prag.

(Vorgelegt in der Sitzung am 9. Juli 1903.)

Nach den Untersuchungen von Goldschmiedt und Knöpfer ${ }^{1}$ kondensieren sich die beiden im Titel genannten Substanzen sowohl unter dem Einflusse gasförmiger Salzsäure als auch unter jenem von wässeriger Kalilauge. In dem ersteren Falle wurde als Kondensationsprodukt ein chlorhaltiges Keton, Chlorbenzyldibenzylketon (1,3, 4-Triphenyl-Chlor-4-butanon-2)

$$
\begin{aligned}
& \mathrm{C}_{6} \mathrm{H}_{5} \cdot \mathrm{CH}-\mathrm{CO} \cdot \mathrm{CH}_{2} \cdot \mathrm{C}_{6} \mathrm{H}_{5} \\
& \mathrm{C}_{6} \mathrm{H}_{5} \cdot \mathrm{CHCl} \text {, }
\end{aligned}
$$

im letzteren zwei Ketole und zwar 1,2,4,5-Tetraphenyl-pentanon-3-diol-1, 5 (I) und 1, 3, 4-Triphenylbutanon-2-ol-4 (II)

I.<smiles>OC(c1ccccc1)C(c1ccccc1)C(O)c1ccccc1</smiles>

II.<smiles>O=C(Cc1ccccc1)C(O)c1ccccc1</smiles>

erhalten.

Die Struktur des chlorhaltigen Ketons war in unzweideutiger Weise durch die Zersetzung festgestellt, die es beim

1 Monatshefte für Chemie, 19, 421 (1898) und 20, 734 (1899). 
Erhitzen auf $160^{\circ}$ erleidet, wobei es glatt in Stilben und Phenylessigsäurechlorid zerfällt, während das Hauptkondensationsprodukt, welches durch Kalilauge entsteht, nur durch die Beobachtung auf obige Formel I zurückgeführt werden konnte, daß es außerordentlich leicht schon beim Erhitzen in Benzollösung oder auch für sich unter Abspaltung von einem Molekül Benzaldehyd sich in. II umwandelt, welches letztere auch wieder leicht zersetzlich ist und Dibenzylketon nebst Benzaldehyd liefert.

Es war daher wünschenswert, die Formel auch noch durch weitere Tatsachen zu stützen, zunächst, weil die Substanz sich als außerordentlich schwer verbrennbar erwiesen hatte, so daß nur unter ganz besonderen Vorsichtsmaßregeln annähernd stimmende Prozentzahlen bei der Elementaranalyse erhalten werden konnten, weil infolge der leichten Zersetzlichkeit schon in nicht hoch siedenden Lösungsmitteln die ebulioskopischen ${ }^{1}$ Molekulargewichtsbestimmungen $\mathrm{zu}$ wenig befriedigenden Resultaten geführt hatten, schließlich weil ein allerdings nur vorläufig in der Eprouvette ausgeführter Versuch, durch Kochen mit Essigsäureanhydrid ein Acetat zu erhalten, erfolglos verlaufen war.

Wir haben uns nun bemüht, auf anderem Wege Ester der beiden Verbindungen zu gewinnen, was auf dem zu beschreibenden Wege glatt verlaufend gelingt und mit Sicherheit die Richtigkeit obiger Formel I dartut.

\section{Tetraphenylpentanondioldiacetat.}

$4 \mathrm{~g}$ Substanz wurden in $30 \mathrm{~g}$ Essigsäureanhydrid suspendiert, auf Eis gekühlt und langsam $2 \mathrm{~cm}^{3}$ konzentrierte Schwefelsäure einfließen gelassen. Der Niederschlag löst sich schnell vollständig auf, die Flüssigkeit färbt sich dort, wo die Säure einfließt, schwach gelbrot, doch verschwindet diese Färbung sofort beim Umrühren. Nach 24 stündigem Stehen wurde die Flüssigkeit, in welcher sich eine geringe Menge einer krystallisierten Substanz ausgeschieden hatte, in Wasser gegossen,

1 Kryoskopische Molekulargewichtsbestimmungen sind wegen der Schwerlöslichkeit der Substanz ausgeschlossen. 
wodurch eine starke, manchmal nahezu farblose, zuweilen etwas rötlich gefärbte Fällung bewirkt wird, sobald das Anhydrid in Wasser sich gelöst hat. Nun wird filtriert und mit Wasser gründlich gewaschen, dann aus Methylalkohol, in welchem der Körper schon in der Kälte ziemlich leicht löslich ist, unter mäßigem Erwärmen umkrystallisiert. Es scheiden sich beim Verdunsten zarte weiße Nädelchen aus, die nach nochmaliger Krystallisation aus Holzgeist konstant bei 180 bis $181^{\circ}$ schmelzen.

I. $0.1954 g$ Substanz gaben $0.5566 \mathrm{~g}$ Kohlendioxyd und $0 \cdot 1015 \mathrm{~g}$ Wasser.

II. $0 \cdot 1915 \mathrm{~g}$ Substanz gaben $0.5459 \mathrm{~g}$ Kohlendioxyd und $0 \cdot 1023 g$ Wasser.

In 100 Teilen:

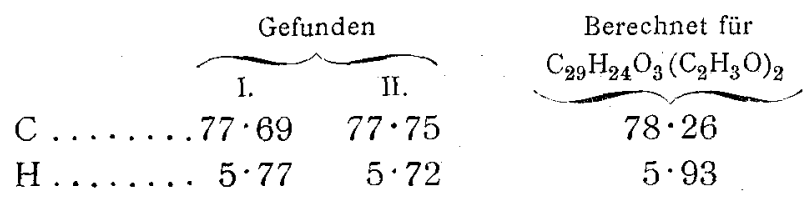

Aus der Mutterlauge konnte eine kleine Menge einer leicht löslichen Substanz gewonnen werden, die sich in konzentrierter Schwefelsäure sehr leicht mit tiefvioletter Farbe löst, während das Acetat dieselben Farbenreaktionen liefert wie das Ketodiol. Wiederholte Versuche, welche den Zweck verfolgten, die Bedingungen für die reichlichere Bildung dieser Substanz ausfindig $z u$ machen, verliefen resultatlos.

\section{Tetraphenylpentanondioldibenzoat.}

Man erhält diesen Ester leicht, wenn man das Ketodiol $(4 g)$ in Pyridin $(50 g)$, worin es sich sehr leicht löst, einträgt und in die in Eis gekühlte Flüssigkeit Benzoylchlorid $(3 g)$ langsam zutröpfelt. Nach 24 Stunden wurde die Flüssigkeit in verdünnte Schwefelsäure, in welcher sich Eisstücke befanden, gegossen, wodurch eine weiße krystallinische Substanz zur Abscheidung kam; manchmal schied sich eine klebrige Masse aus, die, mit kaltem Wasser und dann mit Alkohol verrieben, erstarrte. Aus Methylalkohol wird die Substanz in weißen glänzenden Nädelchen erhalten, die sofort rein sind; die zweite 
Fraktion hatte denselben Schmelzpunkt, $136^{\circ}$, wie die erste. Gegen konzentrierte Schwefelsäure verhält sich die Substanz wie das Ketodiol, aus welchem sie dargestellt worden ist.

$0 \cdot 1769 \mathrm{~g}$ Substanz gaben $0 \cdot 5314 \mathrm{~g}$ Kohlendioxyd und $0 \cdot 0884 \mathrm{~g}$ Wasser.

In 100 Teilen:

$\begin{array}{ccc}\mathrm{C} \ldots \ldots & \begin{array}{c}\text { Gefunden } \\ \mathrm{H} \ldots \ldots\end{array} \quad \frac{51 \cdot 90}{5.54} \quad \frac{\begin{array}{c}\text { Berechnet für } \\ \mathrm{C}_{29} \mathrm{H}_{24} \mathrm{O}_{3}\left(\mathrm{C}_{7} \mathrm{H}_{5} \mathrm{O}\right)_{2}\end{array}}{81 \cdot 90} \\ 5 \cdot 39\end{array}$

Wir haben in gleicher Weise auch Acetat und Benzoat des 1, 3, 4-Triphenylbutanon-2-o1-5 dargestellt:

Triphenylbutanonolacetat. $2 g$ Substanz wurden in Essigsäureanhydrid suspendiert, welches bei Winterkälte $\left(-10^{\circ}\right)$ im Freien gestanden hatte, und einige Tropfen einer ebenfalls gekühlten Lösung von konzentrierter Schwefelsäure in Essigsäureanhydrid hinzugefügt, worauf sofort klare Lösung unter schwacher Gelbfärbung eintrat. Nach 24 Stunden wurde in Wasser gegossen. In dem Maße, als sich das Anhydrid löst, scheidet sich eine feste, rosarot gefärbte Masse aus. Nach wiederholtem Umkrystallisieren aus Methylalkohol erhält man das Acetat in Gestalt schöner büschelförmig angeordneter Nadeln, die konstant bei 109 bis $111^{\circ}$ schmelzen.

$0.1539 g$ Substanz gaben $0.4508 g$ Kohlendioxyd und $0.0820 \mathrm{~g}$ Wasser.

In 100 Teilen:

\begin{tabular}{|c|c|c|}
\hline & Gefunden & $\mathrm{C}_{22} \mathrm{H}_{19} \mathrm{O}_{2}\left(\mathrm{C}_{2} \mathrm{H}_{3} \mathrm{O}\right)$ \\
\hline & $79 \cdot 90$ & $80 \cdot 45$ \\
\hline & $5 \cdot 92$ & $6 \cdot 14$ \\
\hline
\end{tabular}

Triphenylbutanonolbenzoat. Die Darstellung wurde ebenfalls in Pyridinlösung mit Benzoylchlorid unter Eiskühlung vorgenommen, nach 24 Stunden wurde die Flüßigkeit in verdünnte, mit Eis versetzte Schwefelsäure gegossen und das ausgeschiedene Produkt durch Krystallisation aus Methylalkohol gereinigt. Die Substanz schmilzt bei 147 bis $149^{\circ}$. 
$0.1942 \mathrm{~g}$ Substanz gaben $0.5868 \mathrm{~g}$ Kohlendioxyd und $0 \cdot 1003 \mathrm{~g}$ Wasser.

In 100 Teilen:

\begin{tabular}{|c|c|c|}
\hline . & Gefunden & $\begin{array}{c}\text { Berechnet für } \\
\mathrm{C}_{22} \mathrm{H}_{19} \mathrm{O}_{2}\left(\mathrm{C}_{7} \mathrm{H}_{5} \mathrm{O}\right)\end{array}$ \\
\hline $\mathrm{C}$ & $82 \cdot 44$ & $82 \cdot 85$ \\
\hline $\mathrm{H}$. & $5 \cdot 73$ & $5 \cdot 71$ \\
\hline
\end{tabular}

Bei den zahlreichen Kondensationen, welche in den letzten Jahren zwischen Ketonen und Aldehyden von verschiedenen Forschern ausgeführt worden sind, wurden entweder ungesüttigte Verbindungen oder 'Tetrahydropyrone ${ }^{1}$ erhalten; soviel wir aus der Literatur konstatieren konnten, ist nur in einem Falle die Kondensation in dem Stadium des wohl immer, wie allgemein angenommen wird, intermediär gebildeten Aldolkörpers stehen geblieben und zwar bei der Einwirkung von Salzsäure auf ein Gemisch von Acetondicarbonsäure und Paranitrobenzaldehyd, welche, wie Petrenko Kritschenko ${ }^{2}$ gezeigt hat, zu einer Verbindung geführt hat, die unserem Ketole durchaus analog ist. Dieser Körper geht aber schon bei Wasserbadtemperatur unter Kohlensäureverlust in ein ungesättigtes Keton über, während es uns nicht gelungen ist, unserem Ketodiol Wasser zu entziehen; weder das Tetrahydropyron noch das ungesättigte Keton konnte daraus erhalten werden. Da nun Salzsäuregas, als kondensierendes Mittel angewendet, erfahrungsgemäß nicht nur zur Bildung gesättigter chlorhaltiger Ketone, sondern auch direkt zu ungesättigten Ketonen und Tetrahydropyronen führen kann, haben wir dieses Reagens auch auf ein Gemisch von Dibenzylketon (1 Molekül) und Benzaldehyd (2 Moleküle) mit einem kleinen Überschuß an Aldehyd einwirken lassen.

1 Wir sehen von der Beobachtung Abell's ab (Chem. Soc., 79, 1492 [1900]), nach welcher Phenyläthylketon sich unter gewissen Umständen mit Benzaldehyd aldolartig unter gleichzeitiger Reduktion der Ketongruppe kondensiert. Auch jene Kondensationsprodukte, welche, wie Kosta$\mathrm{necky}$ und Rossbach gefunden haben, unter Beteiligung mehrerer Moleküle des Ketons entstehen, können hier außer Betracht bleiben, da hiebei aldolartige Substanzen nicht möglich sind.

2 Berl. Ber., 31, 1510 (1898). 
In ein Gemenge von $10 \mathrm{~g}$ Dibenzylketon und $12 \mathrm{~g}$ Benzaldehyd wurde so lange trockenes Salzsäuregas eingeleitet, bis die Gewichtszunahme an Chlorwasserstoff der theoretischen Menge von 2 Molekülen $\mathrm{HCl}$ entsprach; hierauf wurde das rot gefärbte, breiige Reaktionsgemisch durch 48 Stunden im geschlossenen Gefäße sich selbst überlassen und hierauf ins Vakuum über Kalk gestellt. Mit wenig Methylalkohol konnte der Masse ein gelbes Öl entzogen werden, welches stark nach Benzaldehyd roch. Die Substanz erwies sich als chlorfrei und war in Methylalkohol auch in der Hitze nicht gerade leicht löslich. Sie krystallisierte aus diesem Lösungsmittel beim Erkalten in schönen Prismen. Dieselben zeigten im Schmelzpunktsbestimmungsapparate bei $156^{\circ}$ Beginn des Erweichens, bei $162^{\circ}$ vollständige Verflüssigung.

$0 \cdot 1800 \mathrm{~g}$ Substanz gaben $0.5891 \mathrm{~g}$ Kohlendioxyd und $0.0915 \mathrm{~g}$ Wasser.

In 100 Teilen:

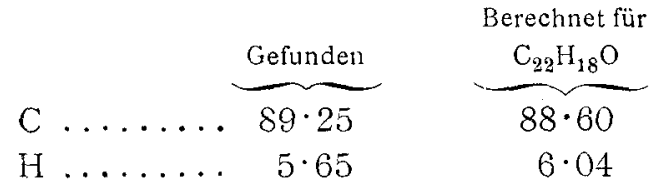

Die Molekulargewichtsbestimmung, ausgeführt nach der Gefriermethode, führte zu folgenden Resultaten:

$0 \cdot 4918 g$ der Substanz bewirkten, in $22 \cdot 4 g$ Benzol gelöst, eine Depression des Gefrierpunktes von $0.365^{\circ}$.

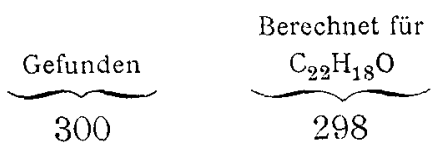

Die Substanz erwies sich nach Zusammensetzung, Molekulargewicht, Schwefelsäurereaktion, Schmelzpunkt als identisch mit dem bei $163^{\circ}$ schmelzenden ungesättigten Keton, das aus dem durch Salzsäure erhaltenen chlorhaltigen Kondensationsprodukte neben anderen Substanzen bei der Einwirkung von Kalilauge erhalten worden ist und der Formel 


$$
\begin{aligned}
& \mathrm{C}_{6} \mathrm{H}_{5} \cdot{ }_{\|}^{\mathrm{C}} \cdot \mathrm{CO} \cdot \mathrm{CH}_{2} \cdot \mathrm{C}_{6} \mathrm{H}_{5} \\
& \mathrm{C}_{6} \mathrm{H}_{5} \cdot \mathrm{CH}
\end{aligned}
$$

entspricht.

Das chlorhaltige Keton selbst, welches bei Anwendung äquivalenter Mengen von Dibenzylketon und Benzaldehyd früher erhalten worden ist, konnte jetzt nicht beobachtet werden, desgleichen war auch das gesuchte Tetrahydropyron im Reaktionsprodukte nicht aufzufinden.

Es ist also auf diesem Wege zwar die direkte Darstellung eines ungesättigten Ketons gelungen, jedoch nicht jene des Tetrahydropyrons.

Petrenko Kritschenk $\mathrm{o}^{1}$ hat gezeigt, daß die Richtung der Wasserabspaltung aus dem intermediär gebildeten Hydroxylderivat bestimmt wird durch die gegenseitige Beeinflussung der Substituenten, welche dem Moleküle eine beständige Konfiguration anweist, die entweder die Bildung ungesättigter oder zyklischer Verbindungen begünstigt. In unserem Falle muß dann wohl die aus dieser Beeinflussung resultierende Konfiguration eine solche sein, daß das Molekül für die Wasserabspaltung überhaupt nicht geeignet erscheint.

Im Anschlusse an vorstehendes seien noch einige Beobachtungen mitgeteilt, welche am Chlorbenzyldibenzylketon gemacht worden sind. Goldschmiedt und Knöpfer hatten, als sie Kalilauge auf dieses Keton einwirken ließen, neben einer unter Wanderung einer Benzylgruppe entstehenden Säure das bereits oben erwähnte, bei $162^{\circ}$ schmelzende, ungesättigte Keton isoliert; nur bei einer Operation war statt dieses Ketons ein solches von gleicher Zusammensetzung gebildet worden, das bei $86^{\circ}$ schmilzt und sich durch die intensiv smaragdgrüne Färbung auszeichnet, mit der es sich in konzentrierter Schwefelsäure auflöst. Die Versuche, dieses Keton wieder zu erhalten, waren damals vergebliche gewesen und auch jetzt neuerdings

1 Berl. Ber., 29, 994 (1896). 
wiederholte, haben nicht zu vollkommen befriedigenden Resultaten geführt. Immerhin haben wir bei einer Operation, bei welcher $4 \mathrm{~g}$ des Chlorbenzyldibenzylketons in kaltem Alkohol suspendiert und mit wenigen Tropfen 75 prozentiger Kalilauge versetzt worden waren, eine Ausscheidung erhalten, nachdem die nur wenig erwärmte und schwach rotgelb gefärbte Lösung in Wasser gegossen worden war. Die Ausscheidung bestand in violetten Flocken, aus denen sich durch fraktionierte Krystallisation aus Methylalkohol beide isomeren ungesättigten Ketone isolieren ließen. Das höher schmelzende Isomere ist das schwerer lösliche.

Die mit den geringen Mengen ausgeführten Versuche, die Ketone, welche wohl stereoisomer sind, ${ }^{1}$ ineinander überzuführen, sind bis jetzt nicht von Erfolg begleitet gewesen, sind aber noch nicht als abgeschlossen zu betrachten.

Bemerkenswert ist übrigens, daß Alkalien, selbst in großer Verdünnung und bei niederer Temperatur, chlorwasserstoffentziehend auf Chlorbenzyldibenzylketon wirken.

Wir sind auf diese Tatsache bei dem Versuche aufmerksam geworden, das chlorhaltige Keton mit naszierendem Wasserstoff zu entchloren, wie es Goldschmiedt und Kreczmar ${ }^{2}$ beim Chlorbenzylphenylaceton leicht gelungen ist. Als wir das Keton in methylalkoholischer Lösung bei zirka $30^{\circ}$ unter fortwährendem Durchleiten von Kohlendioxyd mit 30 prozentigem Natriumamalgam behandelten, erhielten wir aus der alkoholischen Lösung eine sehr gute Ausbeute an dem bei $163^{\circ}$ schmelzenden Keton; ${ }^{3}$ es genügte also die geringe Menge Alkali, welches unter solchen Umständen in Lösung gehen konnte, um vollständige Abspaltung des Chlors zu bewirken. $\mathrm{Ja}$, diese Abspaltung erfolgt sogar schon in der Kälte, wenn man eine alkoholische Lösung des Ketons, genau mit der theoretischen Menge Alkali, in wässeriger Lösung versetzt, stehen läßt. Schon nach kurzer Zeit sind in der Lösung Chlorionen nachweisbar und durch

1 Vergleiche die Beobachtungen von Stob be und Nieden $\mathrm{z}$, Berl. Ber., 34,3904 (1901).

2 Monatshefte für Chemie, 22, 659 (1901).

3 Die Substanz ergab bei der Analyse und Molekulargewichtsbestimmung nachstehende Resultate: 
728 G. Goldschmiedtu. K. Spitzauer, Kondensationsprodukte etc.

Titration eines aliquoten Teiles der Lösung wurde festgestellt, daß die Zersetzung quantitativ verlaufen war.

$0.1956 \mathrm{~g}$ gaben $0.6370 \mathrm{~g}$. Kohlendioxyd und $0.1006 \mathrm{~g}$ Wasser.

In 100 Teilen:

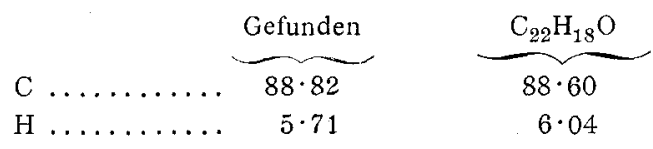

I. $0.1643 \mathrm{~g}$ Substanz in $21.54 \mathrm{~g}$ Benzol zeigten eine Depression $=0.14^{\circ}$.

II. $0.2074 \mathrm{~g}$ Substanz in $17.5 \mathrm{~g}$ Benzol zeigten eine Depression $=0.205^{\circ}$.

$\overbrace{\text { I. }}^{\text {Gefunden }} \underbrace{\text { Berechnet für }}_{\text {II. }} \underbrace{\mathrm{C}_{22} \mathrm{H}_{18} \mathrm{O}}_{298}$

